\title{
Faster swap edge computation in minimum diameter spanning trees
}

\section{Report}

Author(s):

Gfeller, Beat

Publication date:

2012

Permanent link:

https://doi.org/10.3929/ethz-a-006821802

Rights / license:

In Copyright - Non-Commercial Use Permitted

Originally published in:

Technical Report / ETH Zurich, Department of Computer Science 597 


\title{
Faster Swap Edge Computation in Minimum Diameter Spanning Trees
}

\author{
ETH Technical Report 597
}

Beat Gfeller

Institute of Theoretical Computer Science, ETH Zurich, Switzerland

gfeller@inf.ethz.ch

\begin{abstract}
In network communication systems, frequently messages are routed along a minimum diameter spanning tree (MDST) of the network, to minimize the maximum travel time of messages. When a transient failure disables an edge of the MDST, the network is disconnected, and a temporary replacement edge must be chosen, which should ideally minimize the diameter of the new spanning tree. Preparing for the failure of any edge of the MDST, the all-best-swaps (ABS) problem asks for finding the best swap for every edge of the MDST. Given a 2-edgeconnected weighted graph $G=(V, E)$, where $|V|=n$ and $|E|=m$, we solve the ABS problem in $O(m \log n)$ time and $O(m)$ space, thus considerably improving upon the decade-old previously best solution, which requires $O(n \sqrt{m})$ time and $O(m)$ space, for $m=o\left(n^{2} / \log ^{2} n\right)$.
\end{abstract}

\section{Introduction}

For communication in computer networks, often only a subset of the available connections is used to communicate at any given time. If all nodes are connected using the smallest possible number of links, the subset forms a spanning tree of the network. When an edge in a communication tree fails, routing information becomes wrong and message transmission is interrupted. For transient failures that are expected to be repaired quickly, the idea of online point-of-failure rerouting has gained popularity recently $[2,4,6,8]$ : Instead of changing a lot of routing information, only one alternative (so-called swap) edge is used to reconnect the disconnected parts of the tree. For the corresponding change in routing information to be fast, a swap edge for each failing edge needs to be readily available, as the result of an earlier computation. Among all possible swap links for a failing edge, one should choose a best swap link, that is, a swap edge which reconnects the two disconnected parts of the tree in such a way that the resulting swap tree is best w.r.t. some objective.

We show in the following that the common computation of all best swaps ( $A B S$ ) has the further advantage of gaining efficiency (against computing swap edges individually), because dependencies between the computations for different failing edges can be exploited.

In this paper, we are interested in using a Minimum Diameter Spanning Tree (MDST) as the communication tree, i.e., a tree that minimizes the largest distance between any pair of nodes, thus minimizing the worst case length of any transmission path, even if edge lengths are not uniform. Consequentially, a best swap edge in our case minimizes the diameter of the resulting swap tree. Interestingly, this choice of swapping against adjusting the entire tree even comes at a moderate loss in diameter: The swap tree diameter is at most a factor of $5 / 2$ larger than the diameter of an entirely adjusted tree [9].

Related Work. During the last decade, the ABS problem has been investigated for spanning trees with various objectives $[1,8,9,11,13,15]$.

Computing all best swaps of a MDST was one of the first swap problems that were studied. In [9], an algorithm for this problem is given which requires $O(n \sqrt{m})$ time and $O(m)$ space, where the given 
underlying 2-edge-connected communication network $G=(V, E)$ has $n=|V|$ nodes and $m=|E|$ edges. For each of the $n-1$ different tree edges, their algorithm uses somewhat augmented topology trees to select $O(\sqrt{m})$ best swap candidates, then evaluates the quality of each of the $O(\sqrt{m})$ candidate swap edges in $O(1)$ amortized time, and selects the best among them. In order to obtain the $O(1)$ amortized time for computing the diameter of the swap tree associated with a given swap edge, information from a preprocessing phase is used, and combined with an inductive computation that uses path compression.

The authors of [11] studied the ABS problem in a single-source shortest paths tree. In these trees, several meaningful objectives for defining the best swap edge exist, for example (i) minimizing the length of the "detour" path around the failing edge, (ii) minimizing the average distance from the source to all other nodes, or (iii) minimizing the maximum distance from the source to all other nodes. For objective (i) and related ones, the ABS problem can be solved in $O(m \alpha(m, n))$ time and $O(m)$ space using the transmuter data structure [14]. This data structure is very helpful for solving the ABS problem whenever two competing swap edges can be compared independently of which tree edge they are to replace. For objective (ii), the transmuter cannot be used in the same way. The ABS problem for this objective can be solved in $O\left(m \log ^{2} n\right)$ time and $O(m)$ space by mapping it to a geometric problem [1]. The ABS problem for objective (iii) can be seen as a special case of computing best swaps in a MDST. It can be solved in $O(n \sqrt{m})$ time and $O(m)$ space [11]. Indeed, the methods we describe in this paper can improve this running time to $O(m \log n)$. Later, the ABS problem was generalized from single-source shortest path trees to multiple sources, and shown that all best swap edges for a Multiple-Sources Routing Tree, where a best swap edge minimizes the sum of the distances from a given set of sources to all nodes in the tree, can be computed in $O(m n)$ time [15].

Some authors have considered the ABS problem in the setting where transient node failures occur, instead of edge failures. In this context, reestablishing connectivity after a single node failure will in general require more than one swap edge $[10,12]$.

The swap edge computation has also been studied in a distributed setting, where each node of the graph is a computing entity, which initially does not know the entire graph but only its direct neighbors, and its adjacent edges of the selected spanning tree. There, the main goal is not to minimize the running time, but to minimize the number of messages sent during the computation $[2,3,6]$.

Contribution. In this paper, we present an algorithm for computing all best swap edges for a MDST, leading to a proof of our

Main Theorem 1. Given a graph $G=(V, E)$ with $n=|V|$ nodes and $m=|E|$ edges, and a Minimum Diameter Spanning Tree $T$ of $G$, all best swap edges of $T$ can be computed in $O(m \log n)$ time and $O(m)$ space.

For $m=o\left(n^{2} / \log ^{2} n\right)$, this significantly improves upon the time complexity of the previously best known solution [9], using $O(n \sqrt{m})$ time and $O(m)$ space, without increasing the space complexity. Our techniques can also be used to solve the $\{r, \max \}$-problem of [11], which asks for all best swap edges in a shortest paths tree, in time $O(m \log n)$ instead of $O(n \sqrt{m})$.

This improvement over the previous bounds is based on two key ingredients: First, partitioning the set of tree edges into two particular sets, and computing their best swap edges separately using two different techniques, and second, utilizing an essential observation (Lemma 2) to simplify the computation of the diameter in a given swap tree. Our new observations allow for a simpler algorithm than the previous; we use only fundamental data structures.

\section{Terminology}

A communication network is a 2-edge-connected, undirected graph $G=(V, E)$, with $n=|V|$ nodes and $m=|E|$ edges. Each edge $e \in E$ has a non-negative rational ${ }^{1}$ length $l(e)$. The length $|\mathcal{P}|$ of a path

\footnotetext{
${ }^{1}$ Assuming the Real-RAM model, our algorithms would also work for non-negative real weights.
} 
$\mathcal{P}=\left\langle p_{1}, \ldots, p_{r}\right\rangle, p_{i} \in V$, is the sum of the lengths of its edges, and the distance $d(x, y)$ between two nodes $x, y$ is the length of a shortest path between $x$ and $y$. Throughout the paper, we are only dealing with simple paths. The following notation is illustrated in Fig. 1 . Given a spanning tree $T=\left(V, E_{T}\right)$

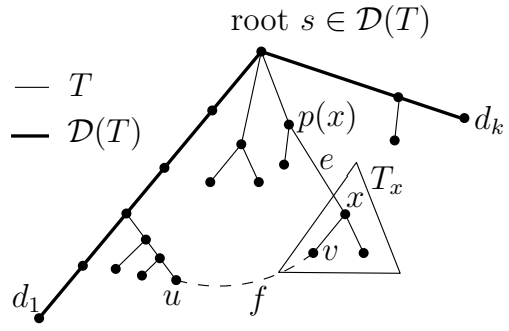

Fig. 1. A MDST $T$ rooted at a node $s$ on its diameter $\mathcal{D}(T)$, a failing edge $e$, and a swap edge $f=(u, v)$ for $e$. The bold line segments denote the diameter $\mathcal{D}(T)$ of $T$.

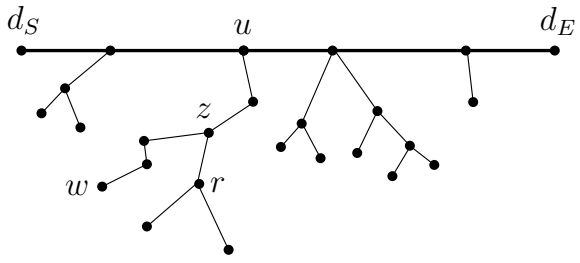

Fig. 2. Illustration for Lemma 2.

of $G$, let $\mathcal{D}(T):=\left\langle d_{1}, d_{2}, \ldots, d_{k}\right\rangle$ denote a diameter of $T$, that is, a longest path in $T$. From now on, we measure distances in the given spanning tree $T$, not in the underlying graph $G$ itself. $T$ is said to be a minimum diameter spanning tree (MDST) of $G$ if it has minimum diameter among all the spanning trees of $G$. For the rest of the paper, we assume that $T$ is a particular MDST of $G$. If we consider $T$ to be rooted at a node on the diameter, say $s$, then for each node $x \neq s$, let node $p(x)$ be the parent of $x$ and $C(x)$ the set of its children. Furthermore, let $T_{x}=\left(V\left(T_{x}\right), E\left(T_{x}\right)\right)$ be the subtree of $T$ rooted at $x$, including $x$.

The removal of any edge $e \in E_{T}$ partitions the spanning tree into two disjoint trees. A swap edge $f$ for $e$ is any edge in $E \backslash E_{T}$ that (re-)connects the two trees, i.e., for which $T_{e / f}:=\left(V,\left(E_{T} \backslash\{e\}\right) \cup\{f\}\right)$ is a spanning tree of $G-e=(V, E \backslash\{e\})$. We denote by $\mathcal{P}_{e / f}$ a longest path in $T_{e / f}$ that goes through swap edge $f$.

Let $F(e)$ be the set of swap edges for $e$. A best swap edge (with respect to the diameter) for $e$ is any edge $f \in F(e)$ for which $\left|\mathcal{D}\left(T_{e / f}\right)\right|$ is minimum. The all-best-swaps (ABS) problem for a MDST is the problem of finding for every edge $e \in E_{T}$ a best swap edge. Throughout the paper, let $e$ denote a failing edge and $f$ a swap edge.

\section{The Quality of a Swap Edge $f$ for a Failing Edge $e$}

We start with a number of observations, all of which are used in our algorithm. Our first observation is that if the diameter of $T_{e / f}$ is longer than $|\mathcal{D}(T)|$, then the new diameter must go through $f$. More precisely:

Lemma 1 (Proved in [6]). For a given failing edge e of the MDST T, the length of the diameter of $T_{e / f}$ is $\left|\mathcal{D}\left(T_{e / f}\right)\right|=\max \left\{|\mathcal{D}(T)|,\left|\mathcal{P}_{e / f}\right|\right\}$.

In our algorithm, we always judge swap edges only according to $\left|\mathcal{P}_{e / f}\right|$, instead of $\left|\mathcal{D}\left(T_{e / f}\right)\right|$. This causes no problem because any swap edge $f$ for which $\left|\mathcal{P}_{e / f}\right|<\left|\mathcal{D}\left(T_{e / f}\right)\right|$ is a best swap edge for $e$, since in this case $\left|\mathcal{D}\left(T_{e / f}\right)\right|=|\mathcal{D}(T)|$.

For a given tree $T=\left(V, E_{T}\right)$, and a given node $r \in T$, let $\mathcal{L}(T, r)$ denote the length of a longest simple path in $T$ which starts in node $r$. Note that for $f=(u, v), \mathcal{P}_{e / f}$ is composed of three parts: the longest path in $T-e$ starting in $u$, the longest path in $T-e$ starting in $v$, and the edge $f$ itself. Thus, we have $\left|\mathcal{P}_{e / f}\right|=l(f)+\mathcal{L}(T-e, u)+\mathcal{L}(T-e, v)$. The following lemma shows how to compute $\mathcal{L}(T, r)$ efficiently for any given node $r \in V$. 
Lemma 2. Let $T=\left(V, E_{T}\right)$ be a weighted tree, and let $\mathcal{D}(T)=\left\langle d_{S}, \ldots d_{E}\right\rangle$ be a diameter of $T$ with endpoints $d_{S}$ and $d_{E}$. Then, the length of a longest simple path inside $T$ starting in $r \in V$ is

$$
\mathcal{L}(T, r)=\max \left\{d\left(r, d_{S}\right), d\left(r, d_{E}\right)\right\} .
$$

Proof. In contradiction, assume there exists a node $w \in V$ such that $d(r, w)>\max \left\{d\left(r, d_{S}\right), d\left(r, d_{E}\right)\right\}$. If $r$ is a node on $\mathcal{D}(T)$, it follows that there exists a path of length $d(r, w)+\min \left\{d\left(r, d_{S}\right), d\left(r, d_{E}\right)\right\}>$ $\max \left\{d\left(r, d_{S}\right), d\left(r, d_{E}\right)\right\}+\min \left\{d\left(r, d_{S}\right), d\left(r, d_{E}\right)\right\}=|\mathcal{D}(T)|$ in $T$, a contradiction.

If $r$ is not on $\mathcal{D}(T)$, let $u$ be the node on $\mathcal{D}(T)$ closest to $r$ (see Fig. 2). If $u$ lies on the path from $r$ to $w$, then the above case shows that starting from $u$, no path, including the path leading to $w$, can be longer than $\max \left\{d\left(r, d_{S}\right), d\left(r, d_{E}\right)\right\}$. Hence, $u$ cannot lie on the path from $r$ to $w$. Let $z$ be the node on the path from $r$ to $w$ closest to $u$ (possibly, $z=r$ ). From $d(r, w)>\max \left\{d\left(r, d_{S}\right), d\left(r, d_{E}\right)\right\}$, we have $d(z, w)>\max \left\{d\left(z, d_{S}\right), d\left(z, d_{E}\right)\right\} \geq \max \left\{d\left(u, d_{S}\right), d\left(u, d_{E}\right)\right\}$ and $d(u, w)>\max \left\{d\left(u, d_{S}\right), d\left(u, d_{E}\right)\right\}$. But this implies that the simple path from $w$ to $u$ and further to $d_{S}$ or $d_{E}$, whichever is further from $u$, has length $d(u, w)+\max \left\{d\left(u, d_{S}\right), d\left(u, d_{E}\right)\right\}>2 \cdot \max \left\{d\left(u, d_{S}\right), d\left(u, d_{E}\right)\right\} \geq|\mathcal{D}(T)|$, a contradiction.

We now show how, given the endpoints of a diameter of $T$, one can compute $\mathcal{L}(T, r)$ for any given node $r$ in constant time, after a preprocessing step requiring $O(n)$ time. We root the tree $T$ at any node, and augment it with

- a labeling of the nodes which allows to obtain the nearest common ancestor (called nca $(a, b)$ for two nodes $a, b)$ of two given nodes in constant time [7];

- in every node $x$, store its distance to the root, called toRoot $(x)$;

This information allows to compute the distance between two arbitrary nodes $a$ and $b$ in the tree $T$ (and thus $\mathcal{L}(T, r))$ in constant time:

$$
\begin{aligned}
d(a, b) & =d(a, \operatorname{nca}(a, b))+d(b, \operatorname{nca}(a, b)) \\
& =\operatorname{toRoot}(a)+\operatorname{toRoot}(b)-2 \cdot \operatorname{toRoot}(\operatorname{nca}(a, b)) .
\end{aligned}
$$

In our algorithm, we distinguish between failing edges on the diameter, called diameter edges, and failing edges not on the diameter, called non-diameter edges. If the given tree has several diameters, we select one and use the same throughout the algorithm. This guarantees that each edge is either a diameter edge or a non-diameter edge, and that this classification is consistent.

\section{Best Swap Edges for Failing Diameter Edges}

In this section, we show how to compute the best swap edges for all failing edges which lie on the diameter $\mathcal{D}(T)$ in time $O(m \log n)$ and $O(m)$ space.

Due to Lemma 1, a given swap edge $f$ for a failing edge $e$ can be evaluated by computing the lengths of the two longest paths starting at its endpoints. It turns out that these lengths can always be found by only considering paths which visit the diameter:

Lemma 3. Consider a tree $T=\left(V, E_{T}\right)$, a diameter $\mathcal{D}(T)$ of it with endpoints $d_{S}, d_{E}$, a failing edge $e$ on $\mathcal{D}(T)$, and an arbitrary node $r \in V$. Let $u$ be the node on $\mathcal{D}(T)$ closest to $r$. One of the longest paths in $T-e$ starting from $r$ contains the node $u$.

Proof. Assume w.l.o.g. that $r$ lies in the same connected component of $T-e$ as $d_{S}$. The proof resembles the proof of Lemma 2: Assume in contradiction that no longest path in $T$ starting from $r$ contains $u$, and let $\langle r, \ldots, w\rangle$ be such a path. Let $z$ be the node on the path from $r$ to $w$ closest to $u$ (possibly, $z=r)$. We have $d(z, w)>d\left(z, d_{S}\right)$, therefore also $d(u, w)>d\left(u, d_{S}\right)$. This is a contradiction, because the path in $T$ going from $d_{E}$ to $u$ and further to $w$ would then be longer than $|\mathcal{D}(T)|$. 
Due to the above lemma, a longest path starting in any endpoint $r$ of a given swap edge can always be found by first going to the node $u \in \mathcal{D}(T)$ closest to $r$. From there, a longest path may either continue to the end of the diameter $\left(d_{S}\right.$ or $\left.d_{E}\right)$, or cross at least one edge towards the failing edge $e$, and possibly leave the diameter again. Note that going from $u$ towards $d_{S}$, but leaving the diameter again, cannot be longer than continuing until $d_{S}$.

For finding the length of a longest path starting from $u$ efficiently, we compute two values $\mu_{S}\left(d_{i}, d_{i+1}\right)$ and $\mu_{E}\left(d_{i}, d_{i+1}\right)$ for every node $d_{i}, i=1, \ldots, k-1$ on the diameter. By $\mu_{S}\left(d_{i}, d_{i+1}\right)$ (respectively $\left.\mu_{E}\left(d_{i}, d_{i+1}\right)\right)$, we denote the length of a longest path in $T$ starting at $d_{S}\left(d_{E}\right)$ and not crossing the edge $\left(d_{i}, d_{i+1}\right)$. Formally, we have $\left(d_{S}:=d_{1}, d_{E}:=d_{k}\right)$ :

$$
\begin{aligned}
\mu_{S}\left(d_{1}, d_{2}\right) & =\mu_{E}\left(d_{k-1}, d_{k}\right)=0 \\
\mu_{S}\left(d_{i}, d_{i+1}\right) & =\max \left\{\mu_{S}\left(d_{i-1}, d_{i}\right), d\left(d_{S}, d_{i}\right)+h\left(d_{i}\right)\right\} \text { for } i=2,3, \ldots, k-1, \text { and } \\
\mu_{E}\left(d_{i}, d_{i+1}\right) & =\max \left\{\mu_{E}\left(d_{i+1}, d_{i+2}\right), d\left(d_{E}, d_{i+1}\right)+h\left(d_{i+1}\right)\right\} \text { for } i=k-2, k-3, \ldots, 1 .
\end{aligned}
$$

where $h\left(d_{i}\right)$ denotes the length of a longest path starting in $d_{i}$, and not using any edges on the diameter $d_{S}, \ldots d_{E}$. It is easy to see that if $T$ is rooted at a node on the diameter, then $h\left(d_{i}\right)$ can be computed for all $d_{i}$ on the diameter in $O(n)$ time by traversing $T$ in postorder. Thus, the values $\mu_{S}\left(d_{i}, d_{i+1}\right), \mu_{E}\left(d_{i}, d_{i+1}\right)$ can be computed for all $d_{i}$ on the diameter in $O(n)$ time.

The following lemma describes how to efficiently compute the longest path in $T-\left(d_{i}, d_{i+1}\right)$ starting from any node $r$.

Lemma 4. Consider any fixed diameter $d_{S}, \ldots, d_{E}$ of a tree $T$, any node $r$ of $T$, and a failing edge $\left(d_{i}, d_{i+1}\right)$ on the diameter. Let $u$ be the node on the diameter closest to $r$. The length of a longest path in $T-\left(d_{i}, d_{i+1}\right)$ starting in $r$ is given by

$$
d(r, u)+\max \left\{d\left(u, d_{S}\right), \mu_{S}\left(d_{i}, d_{i+1}\right)-d\left(u, d_{S}\right)\right\}
$$

if $r$ lies in the same connected component of $T-\left(d_{i}, d_{i+1}\right)$ as $d_{S}$, and

$$
d(r, u)+\max \left\{d\left(u, d_{E}\right), \mu_{E}\left(d_{i}, d_{i+1}\right)-d\left(u, d_{E}\right)\right\}
$$

otherwise.

Proof. We only prove the case where $r$ lies in the same connected component of $T-\left(d_{i}, d_{i+1}\right)$ as $d_{S}$ (the other is completely symmetric). Lemma 3 shows that a longest path first goes from $r$ to $u$ without loss of generality. We now distinguish two cases, depending on whether a longest path starting from $u$ inside $T-\left(d_{i}, d_{i+1}\right)$ can be achieved by using the edge adjacent to $u$ which leads towards $d_{S}$. In this case, a longest path is clearly the one that goes from $u$ until $d_{S}$ (any other cannot be longer in this case because $d_{S}, \ldots, d_{E}$ is a diameter of $T$ ). Otherwise, there must exist a path in $T-\left(d_{i}, d_{i+1}\right)$ starting in $u$ which is longer than $d\left(u, d_{S}\right)$, and which does not use the edge adjacent to $u$ leading towards $d_{S}$. In this case, the length of such a path is exactly $\mu_{S}\left(d_{i}, d_{i+1}\right)-d\left(u, d_{S}\right)$. Furthermore, in this case a longest path in $T-\left(d_{i}, d_{i+1}\right)$ starting in $d_{S}$ must consequently be longer than $2 d\left(u, d_{S}\right)$, that is, $\mu_{S}\left(d_{i}, d_{i+1}\right)>2 d\left(u, d_{S}\right)$. Hence, in any case the correct length can be expressed as $d(r, u)+\max \left\{d\left(u, d_{S}\right), \mu_{S}\left(d_{i}, d_{i+1}\right)-d\left(u, d_{S}\right)\right\}$.

\subsection{Using virtual swap edges}

For any node $v$, let $\operatorname{nc}(v)$ be the node on the diameter which is closest to $v$ (possibly, $\operatorname{nc}(v)=v$ ). According to Lemma 4, the value $\left|\mathcal{P}_{e / f}\right|$ of a particular swap edge $f=(u, v)$ for any failing edge $e$ on the diameter is one of the following four terms (assuming that $u$ lies in the same component of $T-e$ as $d_{S}$, and $v$ lies in the same component of $T-e$ as $\left.d_{E}\right)$ :

1. $d\left(u, d_{S}\right)+d\left(v, d_{E}\right)+l(f)$

2. $d\left(u, d_{S}\right)+\mu_{E}(e)-d\left(\operatorname{nc}(v), d_{E}\right)+d(v, \mathrm{nc}(v))+l(f)$ 
3. $\mu_{S}(e)-d\left(\mathrm{nc}(u), d_{S}\right)+d(u, \operatorname{nc}(u))+d\left(v, d_{E}\right)+l(f)$

4. $\mu_{S}(e)-d\left(\operatorname{nc}(u), d_{S}\right)+d(u, \mathrm{nc}(u))+\mu_{E}(e)-d\left(\operatorname{nc}(v), d_{E}\right)+d(v, \mathrm{nc}(v))+l(f)$.

Note that in all above terms, the part depending on the failing edge $e$ is independent of $f$. Thus, if two swap edges $f^{\prime}$ and $f^{\prime \prime}$ for edge $e$ are such that their values $\left|\mathcal{P}_{e / f^{\prime}}\right|$ and $\left|\mathcal{P}_{e / f^{\prime \prime}}\right|$ both correspond to the same of the four terms above, then we can omit the terms $\mu_{S}(e)$ and $\mu_{E}(e)$ when comparing their quality, without affecting the comparison.

Furthermore, note that since $\mu_{S}\left(d_{i}, d_{i+1}\right)$ is monotonically increasing in $i$, all the failing edges $\left(d_{i}, d_{i+1}\right)$ for which $\max \left\{d\left(u, d_{S}\right), \mu_{S}\left(d_{i}, d_{i+1}\right)-d\left(u, d_{S}\right)\right\}=d\left(u, d_{S}\right)$ form a connected path, as do all the failing edges for which $\max \left\{d\left(u, d_{S}\right), \mu_{S}\left(d_{i}, d_{i+1}\right)-d\left(u, d_{S}\right)\right\}=\mu_{S}\left(d_{i}, d_{i+1}\right)-d\left(u, d_{S}\right)$ (the same holds for $\left.d_{E}\right)$. Thus, for a given swap edge $f=(u, v)$, the set of diameter edges can be divided into at most three sets, each composing a path, such that for each set, $f$ 's value is defined by a specific one of the four terms above. We denote the endpoints of these paths by $q_{1}, q_{2}, q_{3}, q_{4}$. Note that $q_{1}=\operatorname{nc}(u)$ and $q_{4}=\operatorname{nc}(v)$.

The above observations lead to the idea of introducing virtual swap edges which replace the original swap edges, as follows (see also Fig. 3): A virtual swap edge $f_{i}$ consists of its two endpoints, its type, and its value. The two endpoints define a path on the spanning tree $T$, which is equal to the set of diameter edges for which $f_{i}$ is a swap edge. The type of a virtual swap edge is a number in $1,2,3,4$. By definition, two virtual swap edges can only be compared if they have the same type. The value of a virtual swap edge is a rational number. By construction, the quality of each virtual swap edge for a given failing edge $e$ is identical to the quality of the original swap edge which it is replacing. Each swap edge $f$ is replaced by at most three "virtual" swap edges $f_{1}, f_{2}, f_{3}$ in the following way:

- The endpoints are: $f_{1}=\left(q_{1}, q_{2}\right), f_{2}=\left(q_{2}, q_{3}\right), f_{3}=\left(q_{3}, q_{4}\right)$.

- For every failing edge $e$ on $\mathcal{D}(T)$ such that $f \in F(e)$, exactly one of $f_{1}, f_{2}, f_{3}$ is a swap edge.

- The value of each $f_{i}=(u, v)$ is one of the terms shown above, except for the part depending on $e$. Thus, it is either of

1. $d\left(u, d_{S}\right)+d\left(v, d_{E}\right)+l(f)$,

2. $d\left(u, d_{S}\right)-d\left(\operatorname{nc}(v), d_{E}\right)+d(v, \operatorname{nc}(v))+l(f)$,

3. $d\left(v, d_{E}\right)-d\left(\operatorname{nc}(u), d_{S}\right)+d(u, \operatorname{nc}(u))+l(f)$,

4. $-d\left(\operatorname{nc}(u), d_{S}\right)+d(u, \operatorname{nc}(u))-d\left(\operatorname{nc}(v), d_{E}\right)+d(v, \operatorname{nc}(v))+l(f)$.

The number of the term used to compute this value corresponds to the type of the virtual swap edge $f_{i}$.

Note that although there are four different types of virtual swap edges, each individual (original) swap edge is replaced by at most three different virtual swap edges, whose types are all different. In the following, we assume that a swap edge $f$ is replaced by exactly three virtual swap edges; if fewer virtual swap edges are required, the adaptation of the method we describe is straightforward.

Let us summarize.

Lemma 5. The set of all swap edges can be replaced by at most three times as many virtual swap edges, each having one of four types and a value, such that the quality of two swap edges of the same type can be compared based solely on their values. Moreover, this transformation can be carried out using $O(m \log n)$ time and $O(m)$ space.

Proof. For each swap edge $f$ which may replace a failing edge on the diameter, we sequentially compute the virtual swap edges replacing it, as defined above. To obtain $q_{2}, q_{3}$, one needs to find the node $d_{i^{\prime}}$ for which $\max \left\{d\left(u, d_{S}\right), \mu_{S}\left(d_{i^{\prime}}, d_{i^{\prime}+1}\right)-d\left(u, d_{S}\right)\right\} \neq \max \left\{d\left(u, d_{S}\right), \mu_{S}\left(d_{i^{\prime}-1}, d_{i^{\prime}}\right)-d\left(u, d_{S}\right)\right\}$ and the node $d_{i^{\prime \prime}}$ for which

$\max \left\{d\left(v, d_{E}\right), \mu_{E}\left(d_{i^{\prime \prime}}, d_{i^{\prime \prime}+1}\right)-d\left(v, d_{E}\right)\right\} \neq \max \left\{d\left(v, d_{E}\right), \mu_{E}\left(d_{i^{\prime \prime}-1}, d_{i^{\prime \prime}}\right)-d\left(v, d_{E}\right)\right\}$. Using binary search on the sequence of diameter edges from $\left(d_{1}, d_{2}\right)$ to $\left(d_{k-1}, d_{k}\right)$, this can be easily accomplished in $O(\log n)$ time per swap edge, amounting to $O(m \log n)$ time in total. Then, $q_{2}$ is defined as the node among $d_{i^{\prime}}, d_{i^{\prime \prime}}$ which is closer to $d_{S}$, and $q_{3}$ the other node among $d_{i^{\prime}}, d_{i^{\prime \prime}}$. 
Using the virtual swap edges, we compute, for each of the four types $t \in\{1,2,3,4\}$ separately, the best virtual swap edge for all failing edges on the diameter in $O(m \log n)$ time and $O(m)$ space, with the following simple scanline algorithm:

1. Initialize an empty Heap $H_{t}$. The virtual swap edges which are later inserted into $H_{t}$ are to be (heap-)ordered by their values.

2. Consider all failing edges $e_{i}=\left(d_{i}, d_{i+1}\right)$ on the diameter sequentially, for $i=1,2, \ldots, k$. For each $e_{i}=\left(d_{i}, d_{i+1}\right)$ :

- add to $H_{t}$ all those virtual swap edges whose left endpoint (i.e., the one closer to $d_{1}$ ) is $d_{i}$.

- remove from $H_{t}$ all those virtual swap edges whose right endpoint is $d_{i}$.

- store the current minimum of $H_{t}$ as best $\left(e_{i}, t\right)$.

Then, for each $e_{i}$ and each type $t$, replace the virtual swap edge best $\left(e_{i}, t\right)$ by its corresponding swap edge. This yields at most four potential best swap candidates for each diameter edge $e_{i}$, among which a best swap is contained. The best swap edges are then found in time $O(n)$ by simply computing $\left|\mathcal{P}_{e / f}\right|$ explicitly (and in constant time) for each of these selected $O(n)$ candidates.

As we show below, replacing each and every swap edge by its virtual swap edges requires $O(m \log n)$ time and $O(m)$ space, and increases the number of swap edges by a factor of at most three. Summarizing, we have:

Theorem 2. All best swap edges for failing edges on a chosen diameter can be computed in $O(m \log n)$ time and $O(m)$ space $^{2}$.

\section{$5 \quad$ Swap Edges for Failing Non-Diameter Edges}

In this section, we describe an algorithm to compute the best swap edges for those tree edges which do not lie on the chosen diameter $\mathcal{D}(T)$ of the given MDST $T$. We will show that this algorithm runs in $O(m \log n)$ time and requires $O(m)$ space.

For our approach, we root $T$ in an arbitrary node on the diameter, and label all edges by their occurrence in a postorder traversal.

To begin, let $f=(u, v)$ be an edge in $E \backslash E_{T}$ such that $u$ is a descendant of $v$ in $T$, and such that the path from $u$ to $v$ in $T$ does not contain any edge of the diameter $\mathcal{D}(T)$. We call such an edge a backedge. For a backedge $f=(u, v)$, we call the endpoint $u$ the lower endpoint of $f$, and $v$ the upper endpoint of $f$. In the following, we assume for ease of exposition that all edges in $E \backslash E_{T}$ are backedges. In Section 5.6, we describe how to adapt our algorithm to work without this assumption.

Consider the sequence of (non-diameter) tree edges $e_{1}, \ldots, e_{k}$ in the path from $u$ to $v$, starting with the edge adjacent to $u$ : how does $\left|\mathcal{P}_{e_{i} / f}\right|$ depend on the $e_{i}$ ? Since the failing edge $e_{i}$ is not on the diameter $\mathcal{D}(T)$, the connected component of $T-e_{i}$ containing $v$ still contains $\mathcal{D}(T)$. According to Lemma 2, the longest path in $T-e_{i}$ starting in $v$ is therefore the same for all edges $e_{1}, \ldots, e_{k}$, and thus $\mathcal{L}\left(T-e_{i}, v\right)=\mathcal{L}(T, v)$.

On the other hand, the longest path in $T-e_{i}$ starting in $u$ may be different for different failing edges $e_{i}$. To characterize the structure of these paths, we introduce a new concept: The midpoint edge of a tree's diameter is the edge on the diameter which contains the center of the diameter. More precisely, this is the diameter edge whose removal splits it into two parts whose difference in length is minimum (there could be two edges satisfying this definiton; any of them can be chosen). Note that the position of the midpoint edge determines in which direction a longest path starting in a particular node goes (again using Lemma 2): all longest paths which start on one side of the midpoint edge go to the opposite end of the diameter.

\footnotetext{
${ }^{2}$ With a transmuter data structure, a running time of $O(m \alpha(m, n))$ could be achieved here. However, the algorithm in Section 5 requires $\Omega(m \log n)$ time, and hence the asymptotic running time of the complete algorithm would not decrease.
} 


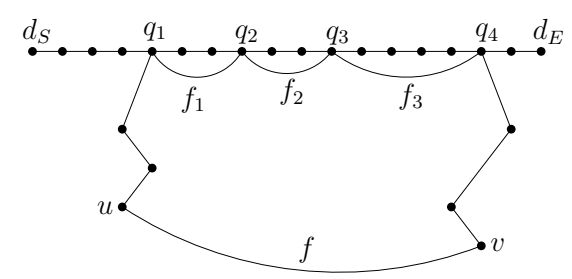

Fig. 3. Replacing a swap edge $f$ by three virtual swap edges $f_{1}, f_{2}, f_{3}$.

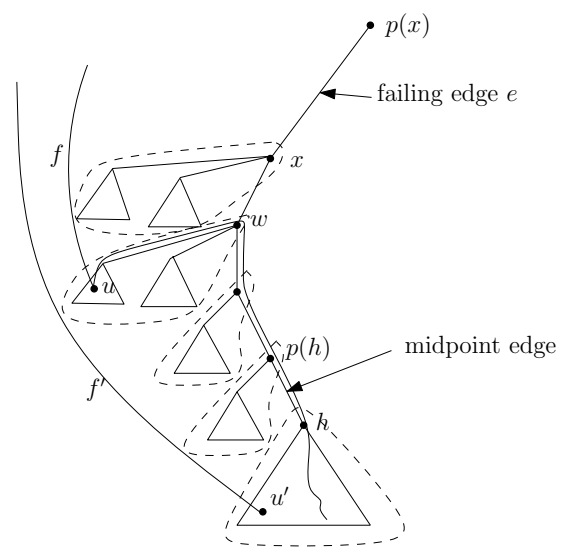

Fig. 4. Grouping of swap edges according to their endpoints in $T_{x}$. Sets of endpoints whose swap edges are grouped together are enclosed in dashed shapes.

We now focus on a particular failing (non-diameter) edge $e=(x, p(x))$ for which the best swap is to be computed. Let $(h, p(h))$ be the midpoint edge of $T_{x}$. By Lemma 2, the longest path starting in $u$ inside $T_{x}$ will contain $(h, p(h))$. This fact allows to partition the set $F(e)$ of swap edges for $e$ into groups as follows (see Fig. 4):

- All swap edges having their lower endpoint below the midpoint edge $(h, p(h))$ will have a longest path going up towards this edge, and then further on to the furthest node in $T_{x}$ (this furthest node is the endpoint of the diameter of $T_{x}$ which lies outside of $T_{h}$, and which is precomputed). We call this the lower group, and denote it by $\mathcal{G}_{\text {lower }}(x)$. Formally, $\mathcal{G}_{\text {lower }}(x):=\left\{(u, v) \in E \backslash E_{T} \mid u \in\right.$ $\left.T_{h} \wedge v \notin T_{x}\right\}$.

- All swap edges having their lower endpoint $u$ above the midpoint edge (i.e., not in $T_{h}$ ) will have a longest path which first leads to some node on the path from $p(h)$ to $x$, then continue down towards the midpoint edge, and finally going into a deepest leaf in $T_{h}$ (this node is the endpoint of $T_{x}$ 's diameter which lies inside $T_{h}$, which is also precomputed). We can partition these swap edges into groups distinguished by the node $w=\operatorname{nca}(u, h)$, the first node on the path from $p(h)$ to $x$ contained in their longest path in $T-e$ starting in $u$. These groups are called the upper groups, and denoted by $\mathcal{G}_{\text {upper }}(x, w)$. Formally:

$\mathcal{G}_{\text {upper }}(x, w):=\left\{(u, v) \in E \backslash E_{T} \mid u \in T_{x} \backslash T_{h} \wedge \operatorname{nca}(u, h)=w \wedge v \notin T_{x}\right\}$.

This grouping is helpful for computing best swap edges, due to the following fact:

Lemma 6. In any group $\mathcal{G}_{\text {upper }}(x, w)$ or $\mathcal{G}_{\text {lower }}(x)$, a best swap candidate for the failing edge $e=$ $(x, p(x))$ is a swap edge $f=(u, v)$ for which $\mathcal{L}(T, v)+l(f)+\operatorname{toRoot}(u)$ is minimum.

Proof. For swap edges in the lower group, all "longest paths" in $T_{x}$ are identical after crossing the midpoint edge. For each upper group corresponding to some node $w$, all "longest paths" in $T_{x}$ are identical after reaching $w$.

In order to compare the best candidates from different upper groups, an additional offset has to be added to each candidate's value, such that the so-called updated value of a candidate $f$ is exactly equal to $\left|\mathcal{P}_{e / f}\right|$. For $f=(u, v)$ with nca $(u, h)=w$, this updated value is

$$
\mathcal{L}(T, v)+l(f)+\operatorname{toRoot}(u)-\operatorname{toRoot}(w)+d\left(w, v_{\text {deep }}\right),
$$

where $v_{\text {deep }}$ is the endpoint of $\mathcal{D}\left(T_{x}\right)$ in $T_{h}$. For the following, it is useful to denote by $\mathcal{G} \mathcal{R}(x)$ the union of all groups associated with a given edge $e=(x, p(x))$. 


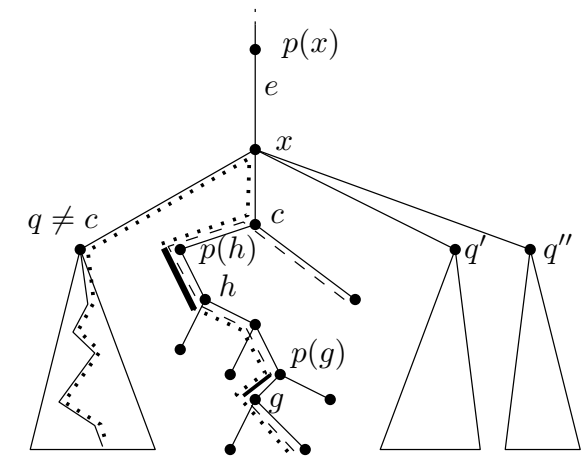

Fig. 5. A failing edge $e=(x, p(x))$, the midpoint edge $(h, p(h))$ of $\mathcal{D}\left(T_{x}\right)$, the child $c \in C(x)$ whose subtree contains $h$, and the midpoint edge $(g, p(g))$ of $\mathcal{D}\left(T_{c}\right)$.

\subsection{Relations between groups for different non-diameter tree edges}

There is a close connection between the midpoint edges of a subtree $T_{x}$ rooted at a node $x$ and the midpoint edges of the subtrees of this node's children. Indeed, the following lemma is easy to prove:

Lemma 7 (Proof omitted). Consider a tree edge $e=(x, p(x))$ and the child $c \in C(x)$ for which the midpoint edge of $\mathcal{D}\left(T_{x}\right)$ is either $(c, x)$ or an edge in $T_{c}$. Then, the midpoint edge of $\mathcal{D}\left(T_{x}\right)$ lies on the path from the midpoint edge of $\mathcal{D}\left(T_{c}\right)$ to e (possibly, the midpoint edges of $\mathcal{D}\left(T_{c}\right)$ and of $\mathcal{D}\left(T_{x}\right)$ are identical).

Thus, the midpoint edge only moves "upwards" when failing edges are visited in postorder: it never occurs that the midpoint edge of $T_{x}$ lies below the midpoint edge of $T_{d}$ for any descendant $d$ of $x$. This implies that for any particular backedge $f=(u, v)$, if a longest path in $T-e_{i}$ starting in $u$ does not visit any child of $u$, then nor will a longest path in any $T-e_{j}, j>i$.

Recall that we consider all (non-diameter) failing edges in a postorder. In the following, we show how the groups of swap edges for a non-diameter tree edge $e=(x, p(x))$ relate to the groups of previously considered failing edges. Later, we exploit these relations using a collection of suitable data structures.

The set of swap edges for edge $e=(x, p(x))$ can be expressed as

$$
F(e)=\operatorname{start}-\operatorname{at}(x) \cup\left\{\bigcup_{q \in C(x)} F((q, x))\right\} \backslash \text { end-at }(x),
$$

where $\operatorname{start-at}(x)$ is the set of swap edges whose lower endpoint is $x$, and end-at $(x)$ is the set of swap edges whose upper endpoint is $x$. We now describe how $F(e)$ is partitioned into the lower group and all the upper groups of $e$.

Let $c \in C(x)$ be the child of $x$ for which the midpoint edge of $\mathcal{D}\left(T_{x}\right)$ is either $(c, x)$ or an edge in $T_{c}$. Furthermore, let $(g, p(g))$ be the midpoint edge of $\mathcal{D}\left(T_{c}\right)$ and let $(h, p(h))$ be the midpoint edge of $\mathcal{D}\left(T_{x}\right)$ (see Fig. 5).

Clearly, all swap edges which belong to the upper group of $e$ associated with $w=x$ are

$$
\begin{gathered}
\mathcal{G}_{\text {upper }}(x, x)=\operatorname{start-at}(x) \cup\left\{\bigcup_{q \in C(x), q \neq c} F((q, x))\right\} \backslash \text { end-at }(x) \\
=\operatorname{start-at}(x) \cup\left\{\bigcup_{q \in C(x), q \neq c} \mathcal{G} \mathcal{R}(q)\right\} \backslash \text { end-at }(x) .
\end{gathered}
$$

For any $w \neq x$ on the path from $p(h)$ to $c$, thanks to Lemma 7, we can express the set of swap edges in the upper group of $e$ associated with $w$ as

$$
\mathcal{G}_{\text {upper }}(x, w)=\mathcal{G}_{\text {upper }}(c, w) \backslash \operatorname{end}-\operatorname{at}(x) .
$$


Finally, the swap edges belonging to the lower group of $e$ are

$$
\begin{gathered}
\mathcal{G}_{\text {lower }}(x)=F((c, x)) \cap\left\{\bigcup_{d \in T_{h}} \text { start-at }(d)\right\} \backslash \text { end-at }(x) \\
=\left(\mathcal{G}_{\text {lower }}(c) \cup\left\{\bigcup_{d \in\langle p(g), \ldots, h\rangle} \mathcal{G}_{\text {upper }}(c, d)\right\}\right) \backslash \text { end-at }(x)
\end{gathered}
$$

\subsection{Our data structure.}

Our approach visits all non-diameter tree edges in postorder, and computes a best swap edge for each of them sequentially. In order to leverage our observations about connections between best swaps for different edges, we associate a data structure, denoted by GroupsDS $(x)$, with each considered edge $e=(x, p(x))$. This structure contains a representation of the group $\mathcal{G}_{\text {lower }}(x)$ and all groups $\mathcal{G}_{\text {upper }}(x, w)$ for $w \in\langle p(h), \ldots, x\rangle$ (recall $(h, p(h))$ denotes the midpoint edge), from which a best swap for $e$ can be extracted in constant time. Moreover, it is designed such that GroupsDS $(x)$ can be efficiently composed of their counterparts of previously visited edges.

Lemma 7 implies that by visiting the (non-diameter) failing edges in postorder results in a corresponding midedge sequence which is also postorder (although this may be only a subset of all tree edges). This is crucial for the correctness of our approach, because once we used a data structure GroupsDS $\left(x^{\prime}\right)$ associated with a previously visited edge $\left(x^{\prime}, p\left(x^{\prime}\right)\right)$, to compute $\operatorname{GroupsDS}(x)$, GroupsDS $\left(x^{\prime}\right)$ is no longer available (as it has been altered/merged into GroupsDS $(x)$ ).

In GroupsDS $(x)$, each group is represented by a Minimum Fibonacci-Heap (short F-Heap in the following). It is widely known that F-Heaps support all of the operations make-heap, insert (.), find-min, merge $(\cdot, \cdot)$ in $O(1)$ amortized time, and the operations delete(.) and delete-min in $O(\log n)$ amortized time, where $n$ is the number of elements in the F-Heap [5].

Each swap edge $f$ contained in a group $\mathcal{G}$ is stored in the corresponding F-Heap, using the value $\mathcal{L}(T, v)+l(f)+\operatorname{toRoot}(u)$, which we call the invariant value of $f$, as its $\mathrm{key}^{3}$. Note crucially, that this value is independent of the failing edge, and according to Lemma 6 the minimum element in the F-Heap corresponding to a group is the best swap (in this group) for the currently considered failing edge. Furthermore, even when this F-Heap is later altered, by inserting or deleting some swap edges, or by merging the F-Heap with another, the value associated with a given swap edge need never be changed. Specifically, GroupsDS $(x)$ contains:

1. the diameter $\mathcal{D}\left(T_{x}\right)$ (given by the labels of its endpoints)

2. the midpoint edge of $\mathcal{D}\left(T_{x}\right):(h, p(h))$ (given by the labels of $h$ and $p(h)$ ). If $x$ is a leaf node in $T$, $h$ is undefined, and in this case, it is assumed that $p(h)=x$.

3 . the child $c \in C(x)$ which contains the midpoint edge $(h, p(h))$. More precisely: either $(c, x)$ is the new midpoint edge, or the midpoint edge is contained in the subtree $T_{c}$ (see Fig. 5).

4. a list heaplist $(x)$ of F-Heaps, containing, for each node $w$ on the path from $p(h)$ to $x$, an F-Heap $\mathrm{FH}_{\text {upper }}(x, w)$ of all swap edges in $\mathcal{G}_{\text {upper }}(x, w)$. The order of the F-Heaps in the list corresponds to the order of the respective nodes $w$ (lowest node first).

5. an F-Heap $\mathrm{FH}_{\text {lower }}(x)$ of all swap edges whose lower endpoint lies in $T_{h}$.

In principle, the best swap for $e$ is found by choosing the candidate with minimum updated value among the best of each group. Doing this naively would require at least linear time in the number of groups, i.e., at least linear in the number of nodes between $e$ and the midpoint edge. To expedite this process during the induction, we use an ordinary Minimum Heap which contains the updated values of the best candidate from each upper group. The best swap edge is then either the minimum element in this heap, or the best candidate from the lower group. Thus, GroupsDS $(x)$ additionally contains the following item:

\footnotetext{
${ }^{3}$ Technically speaking, the key must be made unique by using the tuple $(\mathcal{L}(T, v)+l(f)+\operatorname{toRoot}(u), f)$ as key, where comparisons are based mainly on the first component, and the second is only used to break ties. We omit this detail in the main text for ease of exposition.
} 
6. an ordinary Minimum Heap CandHeap $(x)$, containing for each F-Heap in heaplist $(x)$ the best swap candidate, (heap-)ordered by their quality (i.e., their $\mathcal{P}_{e / f^{-}}$lengths as defined in Equation 1).

From this information, a best swap edge for $(x, p(x))$ is found in constant time by comparing the best candidate in CandHeap $(x)$ with the best candidate in $\mathrm{FH}_{\text {lower }}(x)$, and taking the better of the two:

Lemma 8. Given the data structure GroupsDS $(x)$ associated with the non-diameter tree edge $e=$ $(x, p(x))$, a best swap edge for e can be obtained in constant time.

\subsection{A preprocessing step}

In this section, we explain how to compute the first three items of GroupsDS $(x)$ for all $x$, i.e. the diameter $\mathcal{D}\left(T_{x}\right)$, the midpoint edge of $\mathcal{D}\left(T_{x}\right)$ and the child $c \in C(x)$ containing it, in $O(n)$ time.

First, we traverse all nodes of the tree in postorder, to precompute the following information: In addition to toRoot $(x)$, we associate with every node $x$ the height of its subtree $T_{x}$, called height $(x)$. Second, we compute $\mathcal{D}\left(T_{x}\right)$ for every node $x \in V$. This can be done in $O(n)$ time by traversing the tree in postorder, using induction: the diameter of the subtree rooted at a node $x$ is either equal to the largest diameter found in any of its subtrees, or it is (roughly speaking) a path composed of the longest paths in those two of its subtrees which are deepest when first prolonged by the edge leading to $x$. Furthermore, it is straightforward to associate with each node $x$ the child $c \in C(x)$ which contains the center of $\mathcal{D}\left(T_{x}\right)$.

During the same inductive computation, we associate with every node $x$ the midpoint edge of $\mathcal{D}\left(T_{x}\right)$. All of these midpoint edges can be found in $O(n)$ time as follows: for every non-diameter edge $e_{i}=\left(x_{i}, p\left(x_{i}\right)\right)$ in postorder, find the midpoint edge $m_{i}=\left(h_{i}, p\left(h_{i}\right)\right)$ by considering all non-diameter edges in the same order, and checking for each (in constant time) whether it is the midpoint edge of $\mathcal{D}\left(T_{x_{i}}\right)$. Due to Lemma 7 , we know that either $m_{i}=m_{i-1}$, or $m_{i}$ must come later than $m_{i-1}$ in the given postorder. Thus, we consider each non-diameter edge at most once during this traversal.

\subsection{Our inductive approach}

Let us see how GroupsDS $(x)$ can be constructed efficiently when visiting a new tree edge $e=(x, p(x))$ in the postorder traversal. First, we associate with each node $x \in V$ a list start-at $(x)$ containing all swap edges whose lower endpoint is $x$, and a list end-at $(x)$ containing all swap edges whose upper endpoint is $x$, in $O(m)$ total time.

Lemma 9. In the base case of the induction, when $x$ is a leaf, constructing GroupsDS(e) takes $O(1+$ $\mid$ start-at $(x) \mid)$ amortized time.

Proof. Note that the midpoint edge is undefined in this case, so we assume $p(h)=x$. Hence, $\mathrm{FH}_{\text {lower }}(x)$ is the empty heap, which is constructed in constant time. heaplist $(x)$ contains one entry, namely the F-Heap $\mathrm{FH}_{\text {upper }}(x, x)$, which is created by inserting into an empty F-Heap all edges in start-at $(x)$, using their invariant values for the heap order. This step takes $O(1+|\operatorname{start}-a t(x)|)$ amortized time.

Lemma 10. Consider any tree edge $e=(x, p(x))$, where $x$ is not a leaf node of $T$. Provided that for all children $q \in C(x)$, GroupsDS( $q)$ is available, the data structure GroupsDS(e) can be computed in $O\left(\log n+|C(x)|+(\mid\right.$ start-at $(x)|+|$ end-at $\left.(x) \mid) \cdot \log n+\alpha_{x} \cdot \log n\right)$ amortized time, where $\alpha_{x}=$ $\left(\sum_{q \in C(x)}|\operatorname{heaplist}(q)|\right)-\mid$ heaplist $(x) \mid$.

Proof. Let $c$ be the child of $x$ which contains the midpoint edge of $\mathcal{D}\left(T_{x}\right)$, as computed in Section 5.3. Consider the path $\mathcal{Q}=\langle p(g), \ldots, h\rangle$ from the midpoint edge of $\mathcal{D}\left(T_{c}\right)$ to the midpoint edge of $\mathcal{D}\left(T_{x}\right)$. For the moment, ignore the fact that swap edges in end-at $(x)$ must be removed. Using the relations from Section 5.1, we proceed as follows: $\mathrm{FH}_{\text {upper }}(x, x)$ is obtained by merging all F-Heaps corresponding to a group in $\mathcal{G R}(q)$, for $q \in C(x), q \neq c$, and inserting all edges in start-at $(x)$ 
into the resulting F-Heap, using their invariant values as keys. This step takes $O((\operatorname{start}-a t(x)+$ $\sum_{q \in C(x), q \neq c} \mid$ heaplist $\left.\left.(q) \mid\right) \log n\right)$ amortized time. Similarly, $\mathrm{FH}_{\text {lower }}(x)$ is obtained by merging all FHeaps $\mathrm{FH}_{\text {upper }}(c, d)$, for $d \in\langle p(g), \ldots, h\rangle$, and merging the resulting F-Heap with $\mathrm{FH}_{\text {lower }}(c)$. This step takes $O((\mid$ heaplist $(c)|-|$ heaplist $(x) \mid) \log n)$ amortized time. Still ignoring the swap edges in end-at $(x)$, note that for each $w \in\langle p(h), \ldots, c\rangle$, we have $\mathrm{FH}_{\text {upper }}(x, w)=\mathrm{FH}_{\text {upper }}(c, w)$.

It remains to delete all swap edges in end-at $(x)$ from all these data structures. This can be achieved in $O(\mid$ end-at $(x) \mid \log n)$ amortized time, as follows. For each edge $f=(u, v) \in$ end-at $(x)$, we compute $w=\operatorname{nca}(u, h)$ in constant time, and distinguish two cases: (i) If $w \neq h$, we know by construction that $f$ must be contained in $\mathrm{FH}_{\text {upper }}(x, w)$ (and in no $\left.\mathrm{FH}_{\text {upper }}\left(x, w^{\prime}\right), w \neq w^{\prime}\right)$. We simply remove the edge from this $\mathrm{F}-\mathrm{Heap}^{4}$. (ii) If $w=h$, then by construction $f$ must lie in $\mathrm{FH}_{\text {lower }}(x)$. In this case, we remove $f$ from $\mathrm{FH}_{\text {lower }}(x)$. In both of these cases, if the removed element was the current minimum element of the F-Heap, then the corresponding entry in CandHeap $(x)$ must be deleted, and the new minimum element inserted (unless the F-Heap is now empty).

Due to Lemma 7, heaplist $(x)$ can be obtained from heaplist $(c)$, where all F-Heaps corresponding to nodes on $\mathcal{Q}$ are removed, and adding the new F-Heap $\mathrm{FH}_{\text {upper }}(x, x)$. In CandHeap $(x)$, the candidate corresponding to each of these removed F-Heaps must be removed as well.

Furthermore, the best candidate of the new F-Heap $\mathrm{FH}_{\text {upper }}(x, x)$ must be inserted in CandHeap $(x)$ (unless $\mathrm{FH}_{\text {upper }}(x, x)$ is empty). The exact value for this candidate, say $f$, is $\mathcal{L}(T, v)+l(f)+\operatorname{toRoot}(u)-$ toRoot $(x)+d\left(x, v_{\text {deep }}\right)$, where $v_{\text {deep }}$ is the endpoint of $\mathcal{D}\left(T_{x}\right)$ which lies below the current midpoint edge. Note that $d\left(x, v_{\text {deep }}\right)=l((x, c))+\operatorname{height}\left(T_{c}\right)$.

\subsection{Analysis}

Theorem 3. All best swap edges for failing edges not lying on the chosen diameter can be computed in $O(m \log n)$ time and $O(m)$ space.

Proof. We have already seen in Section 5.3 that the preprocessing step can be completed in $O(n)$ time. It remains to analyze the total cost of all the inductive computations during the postorder traversal.

By Lemmas 9 and 10, the amortized time for visiting a tree edge $(x, p(x))$ is bounded by $O(\log n+$ $|C(x)|+(\mid$ start-at $(x)|+|$ end-at $\left.(x) \mid) \cdot \log n+\alpha_{x} \cdot \log n\right)$ amortized time, where $\alpha_{x}=\left(\sum_{q \in C(x)} \mid\right.$ heaplist $\left.(q) \mid\right)-$ $\mid$ heaplist $(x) \mid$. Furthermore, it is easy to see that

$$
\sum_{x \in V^{\prime}}|\operatorname{end}-\operatorname{at}(x)| \leq m, \quad \sum_{x \in V^{\prime}}|\operatorname{start}-\operatorname{at}(x)| \leq m, \quad \text { and } \quad \sum_{x \in V^{\prime}}|C(x)| \leq n,
$$

where $V^{\prime} \subset V$ is the set of nodes $v$ for which $(v, p(v))$ does not lie on the diameter of $T$. Finally, we have $\sum_{x \in V^{\prime}} \alpha_{x} \leq \sum_{x \in V, x \neq \text { root }} \alpha_{x}=\sum_{x \in V, x \neq \text { root }}\left(\left(\sum_{q \in C(x)} \mid\right.\right.$ heaplist $\left.(q) \mid\right)-\mid$ heaplist $\left.(x) \mid\right)=$ $\left(\sum_{x \in V} \mid\right.$ heaplist $\left.(x) \mid\right)-\sum_{x \in V, x \neq \text { root }} \mid$ heaplist $\left.(x) \mid\right)=\mid$ heaplist $($ root $) \mid$, and since $\mid$ heaplist $(r) \mid \leq n$ for any node $r, \sum_{x \in V^{\prime}} \alpha_{x} \leq n$.

Hence, summing up the time for traversing all tree edges, we obtain $O(n \log n+n+m \log n+$ $m \log n+n \log n)=O(m \log n)$. As to the space complexity, note that at any time during the inductive computation, each swap edge is contained in at most one F-Heap. Therefore, the total amount of space required for these heaps is $O(m)$ at all times. For the other data structures used in our algorithm, the $O(m)$ space bound is obvious.

\subsection{Transforming non-tree edges to backedges}

So far, we have assumed that all swap edges are backedges. We now describe to replace any edge $f$ by at most two "virtual" backedges, whose lengths are defined in such a way that the best swap edge computed by our algorithm is correct. That is, if the computed best swap for a given failing

\footnotetext{
${ }^{4}$ In order to find the element in the given heap in $O(\log n)$ time, a dictionary is required. For simplicity, we omit this detail in the main text.
} 
edge $e$ is a virtual backedge, then replacing the virtual backedge by the edge $f$ it represents yields a (non-backedge) swap edge for $e$ with the same quality. Formally, we replace $f=(u, v)$ by $f_{1}:=\left(u, v_{1}\right)$ and $f_{2}:=\left(v, u_{2}\right)$ (recall that these tuples are ordered), with lengths $l\left(f_{1}\right):=l(f)+d\left(v, v_{1}\right)$ and $l\left(f_{2}\right):=d\left(u, u_{2}\right)$. If the path from $u$ to $v$ in $T$ uses one or more edges of $\mathcal{D}(T)$, we define $v_{1}:=\operatorname{nc}(u)$ and $v_{2}:=\operatorname{nc}(v)$. Otherwise, we define $v_{1}:=\operatorname{nca}(u, v)$ and $u_{2}:=\operatorname{nca}(u, v)$. If it happens that $u=v_{1}$, we omit $f_{1}$, and if $v=u_{2}$, we omit $f_{2}$. To see why this replacement works, note that in both cases $f_{1}$ represents $f$ exactly for all failing edges on the path in $T$ from $u$ to $v_{1}$, and $f_{2}$ represents $f$ exactly for all edges from $v$ to $u_{2}$ (i.e., for each non-diameter tree edge, one of $f_{1}, f_{2}$ represents $f$ ). Furthermore, the lengths of $f_{1}$ and $f_{2}$ are defined exactly such that for any failing edge $e$ for which $f_{i}$ is a swap edge, it holds $\left|\mathcal{D}\left(T_{e / f_{i}}\right)\right|=\left|\mathcal{D}\left(T_{e / f}\right)\right|$.

\section{References}

[1] A. Di Salvo and G. Proietti. Swapping a Failing Edge of a Shortest Paths Tree by Minimizing the Average Stretch Factor. Theor. Comp. Sci., 383(1):23-33, 2007.

[2] P. Flocchini, A. M. Enriques, L. Pagli, G. Prencipe, and N. Santoro. Point-of-failure Shortest-path Rerouting: Computing the Optimal Swap Edges Distributively. IEICE Transactions on Information and Systems, E89-D(2):700-708, 2006.

[3] P. Flocchini, T. M. Enriquez, L. Pagli, G. Prencipe, and N. Santoro. Distributed Computation of All Node Replacements of a Minimum Spanning Tree. In Euro-Par 200\%, volume 4641 of LNCS, pages 598-607. Springer, 2007.

[4] P. Flocchini, L. Pagli, G. Prencipe, N. Santoro, and P. Widmayer. Computing All the Best Swap Edges Distributively. Journal of Parallel and Distributed Computing, 2008. In Press.

[5] M. L. Fredman and R. E. Tarjan. Fibonacci Heaps and Their Uses in Improved Network Optimization Algorithms. J. ACM, 34(3):596-615, 1987.

[6] B. Gfeller, N. Santoro, and P. Widmayer. A Distributed Algorithm for Finding All Best Swap Edges of a Minimum Diameter Spanning Tree. In DISC 200\%, volume 4731 of LNCS, pages 268-282, 2007.

[7] D. Harel and R. E. Tarjan. Fast Algorithms for Finding Nearest Common Ancestors. SIAM Journal on Computing, 13(2):338-355, 1984.

[8] H. Ito, K. Iwama, Y. Okabe, and T. Yoshihiro. Single Backup Table Schemes for Shortest-path Routing. Theor. Comp. Sci., 333(3):347-353, 2005.

[9] E. Nardelli, G. Proietti, and P. Widmayer. Finding All the Best Swaps of a Minimum Diameter Spanning Tree Under Transient Edge Failures. Journal of Graph Algorithms and Applications, 5(5):39-57, 2001. A preliminary version was presented at ESA 1998.

[10] E. Nardelli, G. Proietti, and P. Widmayer. Finding the Most Vital Node of a Shortest Path. Theor. Comp. Sci., 296(1):167-177, 2003.

[11] E. Nardelli, G. Proietti, and P. Widmayer. Swapping a Failing Edge of a Single Source Shortest Paths Tree Is Good and Fast. Algorithmica, 35(1):56-74, 2003.

[12] E. Nardelli, G. Proietti, and P. Widmayer. Nearly Linear Time Minimum Spanning Tree Maintenance for Transient Node Failures. Algorithmica, 40(2):119-132, 2004.

[13] G. Proietti. Dynamic Maintenance Versus Swapping: An Experimental Study on Shortest Paths Trees. In WAE 2000, volume 1982 of $L N C S$, pages 207-217. Springer, 2000.

[14] R. E. Tarjan. Applications of Path Compression on Balanced Trees. J. ACM, 26(4):690-715, 1979.

[15] B. Y. Wu, C.-Y. Hsiao, and K.-M. Chao. The Swap Edges of a Multiple-Sources Routing Tree. Algorithmica, 50(3):299-311, 2008. 